\title{
El Rol de los Jóvenes Profesionales Universitarios en las Organizaciones. Estudio Comparativo Europa/Latinoamérica
}

\author{
Role of young university professionals in organizations. \\ A Europe/Latin-America comparative study
}

\author{
Ángela María Grisales del Rio ${ }^{1}$, Andrés Carrión García ${ }^{1}$, José-Ginés Mora ${ }^{2}$ \\ ${ }^{I}$ Departamento de Estadística e Investigación operativa Aplicadas y Calidad.Centro de Gestión de la Calidad y del \\ Cambio, Universitat Politècnica de València, España. \\ ${ }^{2}$ Institute of Education, London, United Kingdom. \\ angride1@doctor.upv.es \\ acarriondeio.upv.es \\ j.moralioe.ac.uk
}

\begin{abstract}
Resumen - Las exigencias de los puestos de trabajo están evolucionando, y en la actualidad demandan cambios en las competencias profesionales $y$ personales. Estos cambios afectan a aspectos actitudinales, operativos, estratégicos y organizacionales. En este artículo se analiza el papel desarrollado por los profesionales universitarios en las organizaciones en donde trabajan. Para ello se analiza una amplia muestra de jóvenes graduados universitarios de Europa y Latinoamérica. Los resultados muestran que existen entornos económicos y socio-culturales diferentes y que los graduados adaptan su actividad a estos entornos.
\end{abstract}

Palabras clave - Graduados, Organizaciones, Puestos de trabajo, Rol, Universidades.

Abstract - Job requirements have evolved, asking for changes in required professional and personal competencies. These changes affect attitudinal, operative, strategic and organizational aspects. In this paper we analyze the role developed by young university graduates in the organizations where they work. For this purpose a sample of young university graduates in Europe and Latin America are analyzed. The results show the existence of different economic and socio-cultural environment and how young graduates adapt its activity to these environments.

Keyword - Graduates, Jobs, Organizations, Role, Universities.

\section{INTRODUCCIÓN}

En los últimos veinte años se ha producido una transformación en el contexto de las organizaciones, provocando cambios en las estrategias de gestión para garantizar la supervivencia de las mismas [1], [2], [3], [4], [5]. Comprender esta evolución ayuda a entender la evolución paralela que se ha dado en los requerimientos del perfil laboral y profesional [6], [7].
Desde mediados de los años ochenta hasta finales de los noventa del pasado siglo, hubo un cambio de la "cultura del esfuerzo" a la "cultura de control de gestión" en lo que se relaciona con lo que las empresas esperan de su fuerza de trabajo [8].

La globalización ha acelerado los cambios, debido al aumento de la competencia a una dimensión global y de la necesidad de un proceso de reducción de costes para mantener la rentabilidad de las empresas [9].

El papel de los profesionales en las organizaciones se ha transformado, en un intento de adaptarse a estos cambios. Las crisis organizacionales y personales han creado un conflicto que aún no está bien resuelto en muchas organizaciones [10].

Cada organización exige diferentes perfiles profesionales a su personal, de acuerdo con los diferentes roles a desarrollar: habilidades técnicas, actitudes personales, competencias operativas, comprensión de las estrategias corporativas, etc. [11].

En una economía cerrada, la principal característica operativa requerida en el personal era la capacidad de trabajo. Dominaban las estructuras verticales y a las personas se les pedía esfuerzo, dedicación y disciplina [12]. La economía competitiva requiere de los trabajadores algo más que simplemente el obedecer órdenes. Este nuevo entorno requiere pasar del trabajo individual al trabajo en equipo, desde la simple obediencia a compartir ideas e información. La integración y la comunicación son los nuevos valores dominantes. Las habilidades de gestión necesitan ser reforzadas, así como la capacidad de analizar los datos y resolver problemas [11], [13]. 
La globalización requiere nuevos profesionales, que asuman cada vez mayor iniciativa y responsabilidades (empowerment). La autonomía y la integración cultural, la comunicación (incluyendo un aumento en la importancia de las lenguas) y la actualización técnica y profesional permanente son características de esta situación.

El aumento de la virtualización de la economía ha cambiado la forma en que trabajan las personas y su relación con las organizaciones y colegas. Conceptos tradicionales como el de autoridad están cambiando, y las estructuras organizativas se están moviendo a una reticularidad creciente. Las estructuras unidireccionales verticales están demostrando ser insuficientes para esta nueva situación. Las características personales que han adquirido mayor importancia son la capacidad de trabajar en entornos de incertidumbre, la capacidad de trabajar bajo presión, y un alto nivel de implicación personal, aparte de los conocimientos técnicos para el uso de tecnologías de la información [13].

Los cambios en el entorno organizativo y económico han modificado los roles de los diferentes actores presentes en las organizaciones. Las relaciones personales con la organización han cambiado notablemente. El "de por vida" concepto de los años anteriores a los ochenta, típico en grandes organizaciones (públicas y privadas) se ha cambiado a una relación (temporal) basada en el ajuste de la situación personal (habilidades) y los requisitos de la empresa. En algunos casos esto ha creado situaciones precarias difíciles de manejar [14].

\section{A. Objetivos del estudio}

Como resultado de la evolución y los cambios comentados en el apartado anterior, el papel que juegan las personas en las organizaciones está cambiando. En este artículo nos ocupamos de la cuestión de la identificación y cuantificación de cómo los profesionales jóvenes desarrollan su actividad en las organizaciones. Se analizarán las percepciones de los graduados acerca de diferentes aspectos de su posición y su papel en las organizaciones.

El estudio fue desarrollado en diferentes países de Europa y América Latina. Un análisis comparativo como el realizado aumenta nuestra comprensión de su situación.

Las preguntas a responder son: ¿Cuáles son las posiciones de los graduados universitarios en las organizaciones? ¿Cuáles son las funciones que desarrollan en su trabajo? ¿Hay diferencias entre los graduados europeos y latinoamericanos? ¿Qué diferencias?

\section{LOS PROYECTOS REFLEX Y PROFLEX}

Los datos utilizados para este artículo fueron obtenidos en el marco de los proyectos europeos $\operatorname{REFLEX}^{1}$ y PROFLEX $^{2}$

El acrónimo REFLEX corresponde al proyecto titulado "El Profesional Flexible en la Sociedad del Conocimiento. Nuevas exigencias a la Educación Superior en Europa ". En el estudio participaron más de catorce países de Europa. Una encuesta fue dirigida a los graduados europeos cinco años después de su graduación en 1999 - 2000. Las conclusiones obtenidas a partir del análisis de cerca de 38.000 encuestas, se refieren al papel de estos graduados en la sociedad del conocimiento, así como los factores que influyen en el éxito de estos jóvenes profesionales, entre otros aspectos.

El segundo proyecto, PROFLEX, es un Proyecto que replica al REFLEX para universidades y graduados de Latinoamérica. Sus objetivos incluyen la obtención de resultados sobre la experiencia educativa y el empleo de los graduados, cinco años después de haber terminado sus estudios superiores. Como resultado se obtuvo una base datos de unos 10.000 jóvenes profesionales universitarios de diez países de América Latina, graduados en 2002-2003 [15].

Uno de los puntos fuertes de este estudio, radica en el gran número de encuestas utilizadas y el amplia área geográfica cubierta por la investigación, y también en el uso de herramientas estadísticas avanzadas.

\section{METODOLOGIA}

Los datos incluyen resultados de una encuesta a graduados universitarios de 24 países, en posesión de un grado de Educación Superior (no incluye programas de posgrado y doctorado). Las encuestas fueron tomadas cinco años después de la graduación. Las encuestas en catorce países europeos fueron realizadas entre 2005 y 2006, los graduados de los 10 países de Latinoamérica

\footnotetext{
${ }^{1}$ El Proyecto REFLEX fue financiado por el $6^{\text {to }}$ Programa Marco de la UE. http://www.roamaastricht.nl/?portfolio=reflexinternational-survey-higher-education-graduates

${ }^{2}$ El proyecto ALFA_PROFLEX fue financiado por la UE y universidades de Europa y Latinoamérica.

Coordinado por el Centro de Gestión de la Calidad y del Cambio de la Universitat Politècnica de València, España. www.seguimientoegresados.com
} 
fueron encuestados entre 2007 y 2010. Se realizaron un total de 48.094 encuestas, 38.286 de Europa y de Latinoamérica 9.808.

El cuestionario utilizado es completamente paralelo en ambas encuestas, excepto que el europeo incluye una serie de preguntas relacionadas con algunas características de la organización en la que los graduados desarrollan su actividad. Por lo tanto, alrededor de sesenta preguntas conforman el marco común de datos, de los cuales veinte son de interés para este estudio.

\section{A. Estadística Descriptiva}

El primer paso fue la depuración inconsistencias en los datos, para tratar después con la descripción de las principales variables incluidas en el cuestionario. Se obtuvieron estadísticas básicas (máximo y mínimo, media, varianza, asimetría y curtosis ...), así como algunas pruebas de independencia y normalidad.

Las variables de interés fueron: Continente (Europa y América Latina), el cargo ocupado, las variables que describen el nivel de responsabilidad de los graduados en las organizaciones, la autonomía en su actividad, las relaciones establecidas dentro y fuera de la organización y la ética profesional.

Para describir algunas de las respuestas se utilizaron histogramas de frecuencias, así como tablas de frecuencias. Se utilizaron tablas de contingencia, con los test de independencia (test de $\chi^{2}$ ), para obtener una idea sobre la relación entre las variables.

\section{B. Estudio de Correlaciones y Fiabilidad}

Se analizaron las correlaciones entre las variables y se verificó la fiabilidad de las escalas utilizadas.

\section{Análisis Factorial}

Como método de análisis se utilizó el análisis factorial (AF) que es una poderosa técnica estadística multivariante orientada a la reducción de datos, para encontrar relaciones entre las variables utilizadas y para identificar los factores subyacentes en la información de las variables originales. El análisis de componentes principales (ACP) se utilizó en la búsqueda de estos factores subyacentes, para explicar la mayor cantidad posible de la varianza total.

\section{POSICIÓN OCUPADA}

\section{A. Descriptivo}

En ambos proyectos, REFLEX y PROFLEX, la codificación de los puestos de trabajo se realizó utilizando la Clasificación Internacional Uniforme de Ocupaciones (CIUO-88). Esta clasificación se estructura mediante la agrupación de los diferentes puestos de trabajo de acuerdo a la función, nivel de responsabilidad, dificultad y grado de especialización necesario. Sólo el primer dígito se utiliza en la clasificación en este estudio.

La Figura 1 muestra la distribución total de los puestos de trabajo que ocupaban los graduados en el momento de la encuesta (cinco años después de la graduación). Se utilizaron para el análisis 40.124 encuestas válidas y los resultados obtenidos indican que el porcentaje más alto corresponde a cargos de profesional/científico (61\%), seguido de los cargos técnicos y profesionales de nivel medio (19,6\%). En la muestra conjunta, sólo el 9,5\% de los graduados ocupan cargos directivos. Estas cifras son coherentes con la idea de los jóvenes profesionales, en sus primeros años de actividad profesional.

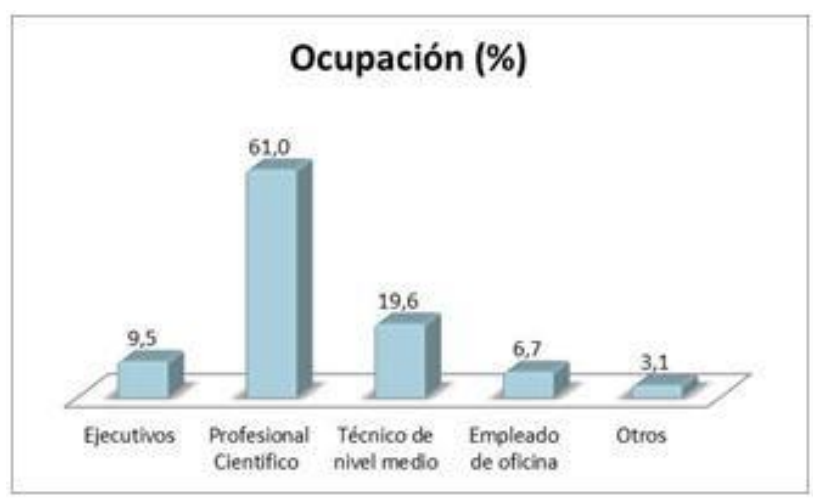

Fig. 1. Cargos ocupados por graduados universitarios

\section{B. Comparación}

La Tabla 1 compara los puestos de trabajo de los graduados europeos y latinoamericanos. En ambos grupos, el puesto de trabajo más frecuente es el Profesional/científico, con el $63,1 \%$ y $51,8 \%$ respectivamente, seguido por los técnicos y profesionales de nivel medio, con un $20,5 \%$ y $16 \%$ respectivamente.

\begin{tabular}{|c|c|c|}
\hline Ocupación & Europa & Latinoamérica \\
\hline Directivos & $8,50 \%$ & $14,10 \%$ \\
\hline $\begin{array}{c}\text { Profesional / } \\
\text { Científico }\end{array}$ & $63,10 \%$ & $51,80 \%$ \\
\hline $\begin{array}{c}\text { Técnico de } \\
\text { nivel medio }\end{array}$ & $20,50 \%$ & $16,00 \%$ \\
\hline $\begin{array}{c}\text { Empleado de } \\
\text { oficina }\end{array}$ & $5,10 \%$ & $13,90 \%$ \\
\hline Otros & $2,80 \%$ & $4,30 \%$ \\
\hline Total (N) & 32572 & 7552 \\
\hline
\end{tabular}


Tabla 1. Ocupación por Continente

La Tabla 2 muestra los resultados obtenidos a partir de la realización de la prueba $\chi^{2}$ para comprobar la independencia de las variables continente y ocupación. La prueba verifica que existe diferencias estadísticamente significativas entre los puestos de trabajo de los graduados europeos y Latinoamericanos.

Aparecen dos diferencias relevantes: Mientras que sólo el $8.5 \%$ de los graduados europeos tienen cargos directivos, este valor aumenta hasta el $14,1 \%$ en los graduados de Latinoamérica. En el otro extremo de la escala, mientras que sólo el 7,9\% de los graduados europeos tienen posiciones de bajo nivel, como empleado de oficina y otros, en los graduados de Latinoamérica este valor es $18,2 \%$.

\begin{tabular}{|c|c|c|c|}
\hline & Valor & gl & $\begin{array}{c}\text { Sig. asintótica } \\
\text { (bilateral) }\end{array}$ \\
\hline $\begin{array}{c}\text { Chi-Cuadrado de } \\
\text { Pearson }(\chi 2)\end{array}$ & 1142,965 & 4 & 0,000 \\
\hline $\begin{array}{c}\text { Razón de } \\
\text { verosimilitudes }\end{array}$ & 1005,682 & 4 & 0,000 \\
\hline $\begin{array}{c}\text { Asociación } \\
\text { lineal por lineal }\end{array}$ & 116,338 & 1 & 0,000 \\
\hline
\end{tabular}

Tabla 2. Test de Independencia para Variables Continente y Ocupación

No es fácil interpretar estos hechos, pero como una posibilidad se puede considerar que la primera diferencia principal puede ser debido al hecho de que en América Latina la educación superior todavía está limitada a una parte de la población con mayor nivel económico, y con frecuencia los estudiantes tienen las relaciones sociales que facilitan alcanzar puestos de dirección a principios de su carrera profesional antes que los graduados europeos. La estratificación social también puede estar en la base para el segundo hecho, los graduados Latinoamericanos procedentes de algunas universidades y entornos socioeconómicos pueden encontrar más dificultades para llegar a los puestos de trabajo de acuerdo con su nivel profesional.

Las diferentes estructuras de los grados universitarios, y las diferencias socioeconómicas también pueden ayudar a explicar la diferente forma de la distribución de los puestos de trabajo.

\section{EL ROL DE LOS JÓVENES GRADUADOS UNIVERSITARIOS}

A. Área de estudios apropiada al trabajo

La variable "Ajuste Horizontal" evalúa el ajuste entre el área de estudio en el que se graduaron los profesionales y el área de conocimiento requerida por su actividad. Mediante una tabla de contingencia se comprueba si este ajuste depende del continente en el que viven. La Tabla 3 presenta los resultados, que muestran que hay diferencias significativas entre los graduados europeos y latinoamericanos para la prueba realizada.

\begin{tabular}{|c|c|c|c|}
\hline & Valor & gl & $\begin{array}{c}\text { Sig. asintótica } \\
\text { (bilateral) }\end{array}$ \\
\hline $\begin{array}{c}\text { Chi-Cuadrado de } \\
\text { Pearson }(\chi 2)\end{array}$ & 202,166 & 3 & 0,000 \\
\hline $\begin{array}{c}\text { Razón de } \\
\text { verosimilitudes }\end{array}$ & 224,863 & 3 & 0,000 \\
\hline $\begin{array}{c}\text { Asociación } \\
\text { lineal por lineal }\end{array}$ & 157,285 & 1 & 0,000 \\
\hline
\end{tabular}

Tabla. 3. Test de Independencia para las Variables Continente y Ajuste Horizontal

A la pregunta "¿cuál es el área de estudios más apropiado para este trabajo?" El 85,1\% de los graduados europeos respondieron que en su trabajo, el área que se requiere es su propia área de conocimiento o alguna relacionada, mientras que para los graduados de Latinoamérica este valor es de $90,8 \%$.

Por el contrario, el $7 \%$ de los graduados europeos consideran que están trabajando en una posición que requiere un campo completamente diferente de los conocimientos que tienen. Para los graduados de Latinoamérica este valor es sólo el 5,2\%. Si incluimos la categoría "Sin campo en particular" valores aumentan hasta el $14,9 \%$ en el caso de Europa y el 9,2\% para los graduados de LA. La Figura 2 muestra la distribución de estas respuestas.

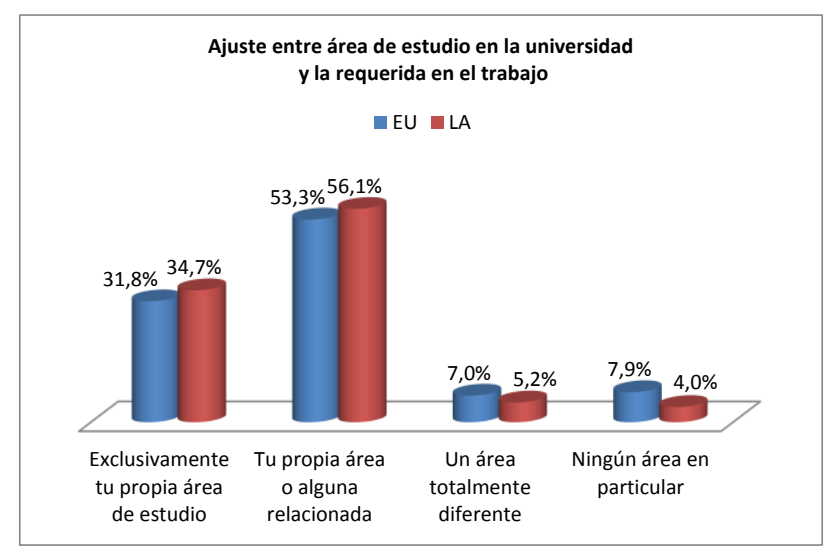

Fig. 2. Ajuste entre el área de estudio en la universidad y la requerida en el trabajo

\section{B. Responsabilidad}

La responsabilidad y autonomía asociadas al puesto de trabajo se han medido con un grupo de preguntas usando una escala de 1 a 5 ( 1 = "Nada" a 5 = "Mucho"). Se les pide a los graduados responder a las siguientes preguntas: 
a) ¿En qué medida es responsable de establecer objetivos para la organización?

b) ¿En qué medida es responsable de establecer objetivos para su propio trabajo?

c) ¿En qué medida es responsable de decidir estrategias de trabajo para la organización?

d) ¿En qué medida es responsable de decidir cómo hace su propio trabajo?

e) ¿Cuánto perjudicaría a la organización si cometiera un error grave en su trabajo?

La figura 3 muestra los resultados obtenidos a partir de las respuestas de ambos grupos para las preguntas citadas anteriormente.

Para la pregunta sobre la responsabilidad de establecer objetivos para la organización, el 37,5\% de los europeos manifestaron no tener ninguna responsabilidad, frente al $13,1 \%$ de los latinoamericanos que hicieron idéntica valoración. La respuesta que indica un alto nivel de responsabilidad es seleccionada por los europeos en el $10 \%$ de los casos, mientras que para los latinoamericanos este valor es del $21 \%$. La Figura 3a muestra los diferentes patrones de respuesta.

Para la pregunta sobre la responsabilidad de establecer objetivos del propio trabajo, el patrón es muy similar, pero los latinoamericanos declaran tener una mayor autonomía. Véase la Figura 3b.

Para la pregunta acerca de la responsabilidad para decidir las estrategias de trabajo para la organización, los resultados de la pregunta a) se repiten: Los Europeos declaran tener una responsabilidad muy limitada, mientras que América Latina declaran tener alto nivel de responsabilidad para establecer estrategias para la organización.

A la pregunta sobre la responsabilidad para decidir cómo hacer el trabajo propio, el patrón de respuestas de la pregunta b) se repite, mostrando similitud entre ambos grupos, pero los latinoamericanos declaran tener mayor autonomía.

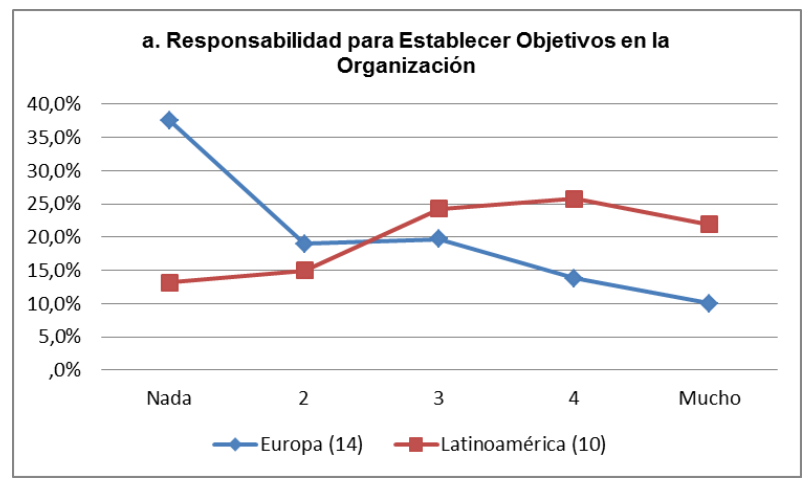

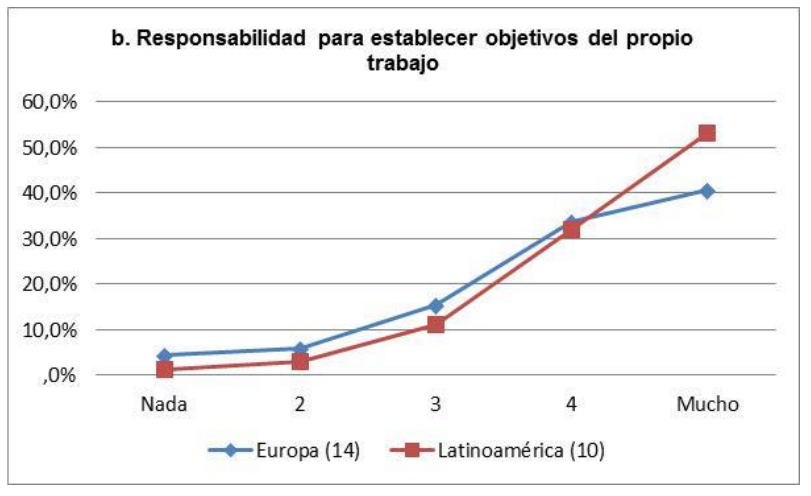
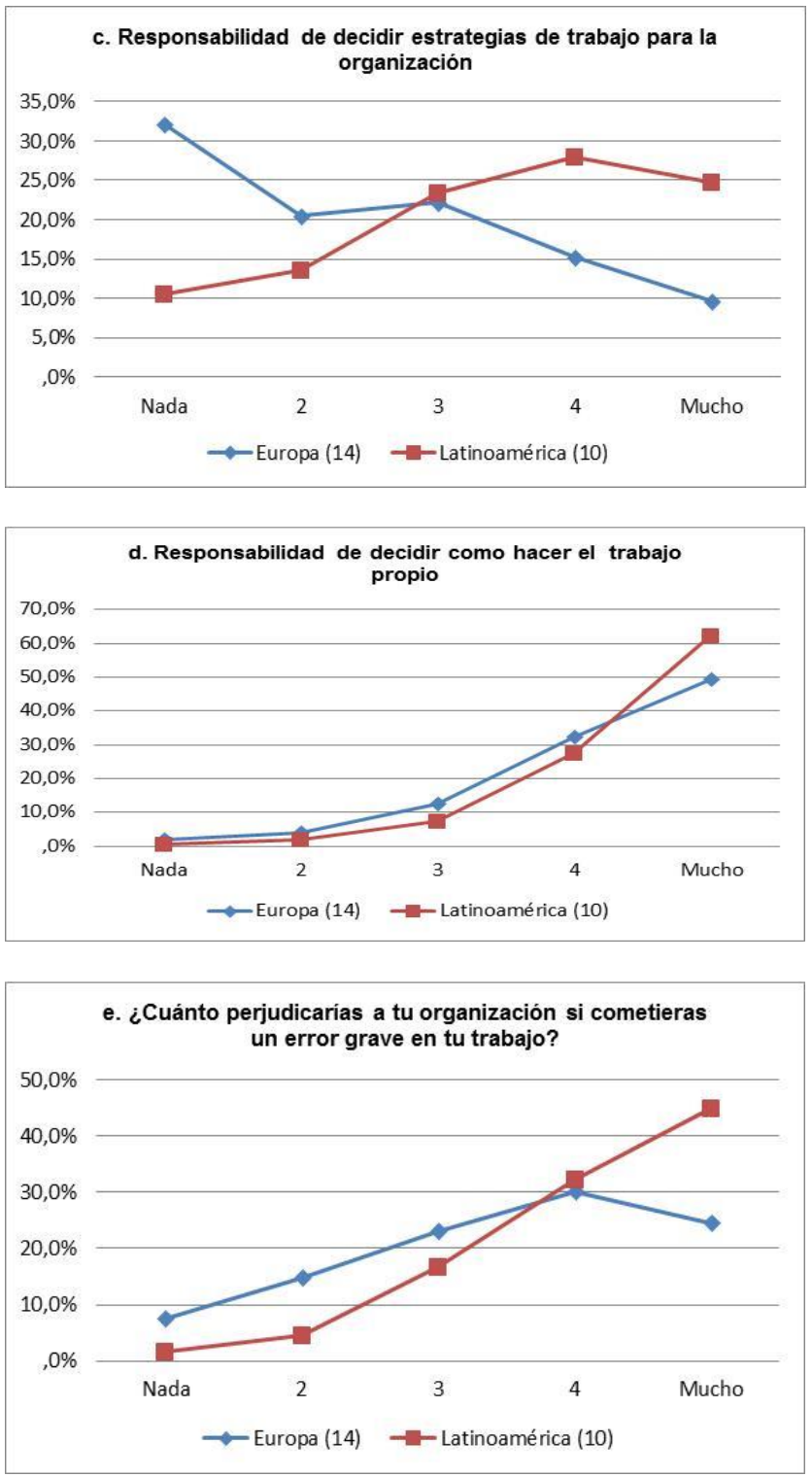

Fig. 3. Responsabilidad y autonomía

Finalmente la pregunta relacionada con el nivel de daño que causaría el graduado si cometiera un error grave en el trabajo, el patrón se acerca al de las preguntas b) y d): de similar forma los latinoamericanos declaran tener un mayor nivel de daño potencial que el manifestado por los 
europeos (en concordancia con su mayor nivel de responsabilidad).

En resumen, como resultado del análisis de estas preguntas, los graduados de Latinoamérica tienen una mayor responsabilidad en el proceso de toma de decisiones de sus organizaciones que sus colegas europeos y en consecuencia, potencialmente pueden causar mayor daño a la organización. Los niveles de autonomía son muy similares en los dos grupos o ligeramente mayor para los graduados de LA. Los resultados mostrados en ñas figuras $3 \mathrm{a}, 3 \mathrm{~b}, 3 \mathrm{c}$ y $3 \mathrm{~d}$ son coherentes con la mayor frecuencia con que los jóvenes profesionales latinoamericanos ocupan puestos directivos.

\section{Relaciones y ética profesional}

Se utilizaron escalas de Likert para un grupo de preguntas que tienen que ver con las relaciones (internas y externas) en el trabajo y con la ética. Las escalas de Likert utilizadas tienen cinco niveles, desde $1=$ "Nada" a $5=$ "Mucho". Se preguntó a los graduados si estaban de acuerdo con las afirmaciones siguientes:

a) Los compañeros, clientes, etc., acuden a mí para pedirme consejo.

b) Mantengo informados a mis compañeros de profesión sobre los nuevos desarrollos en mi campo.

c) Suelo iniciar relaciones profesionales con expertos ajenos a la organización.

d) Importancia de la ética profesional como parte importante de las funciones.

La figura 4 muestra las respuestas para estas cuatro preguntas. Para las afirmaciones a) y b), los patrones de respuestas son similares para los dos grupos, siendo los latinoamericanos quienes declaran ligeramente mayor capacidad de "consulta" que los europeos. En la afirmación c), las diferencias son más claras. La iniciativa de establecer contactos fuera de la organización es significativamente mayor en los graduados de Latinoamérica.

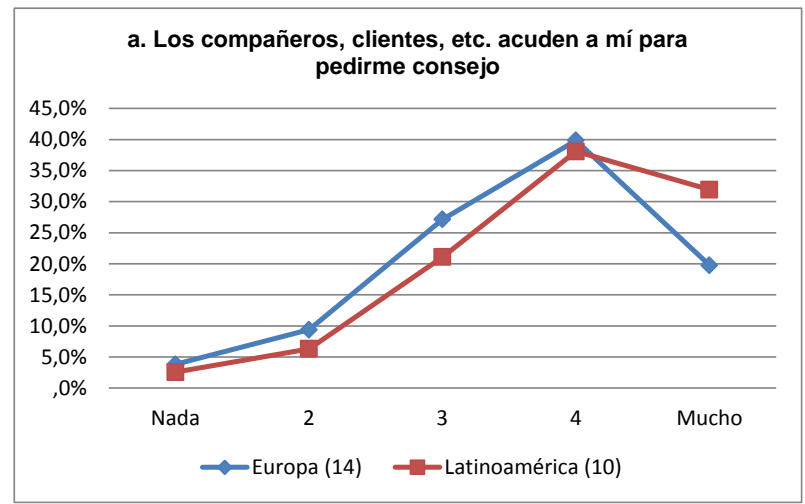

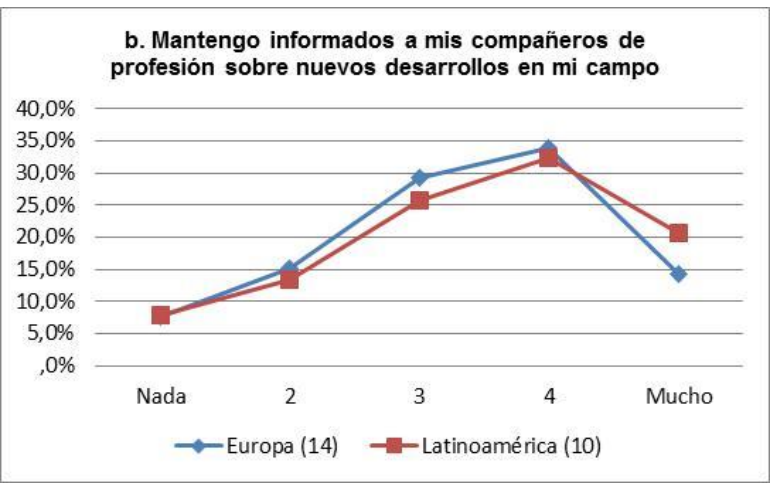
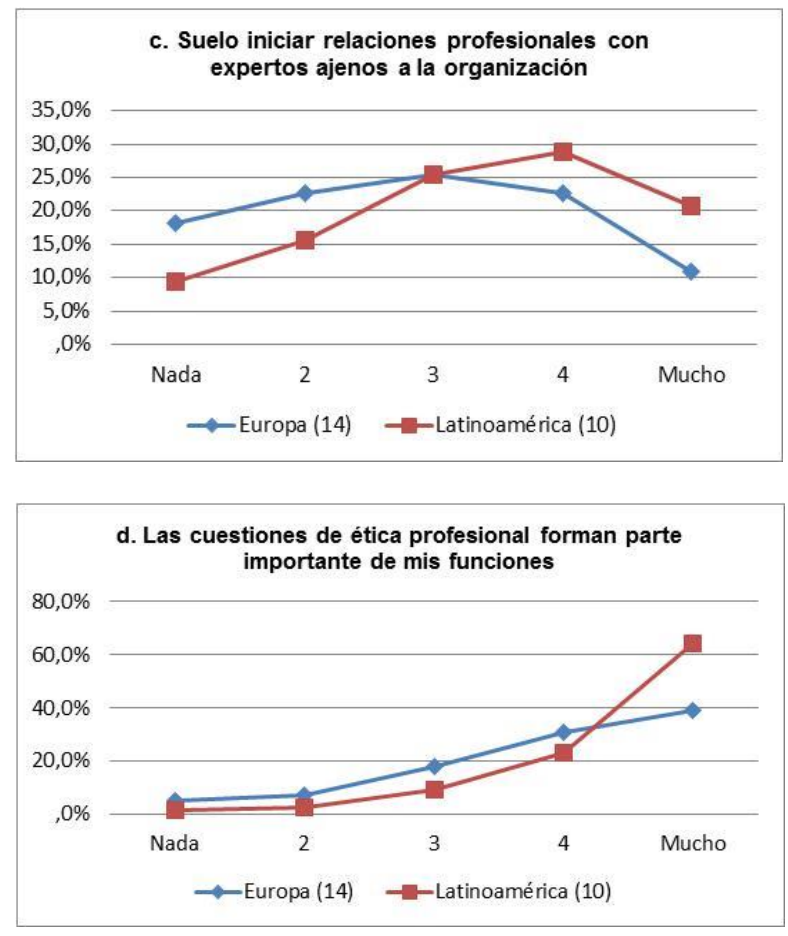

Fig. 4. Relaciones en el trabajo

Por último, para la afirmación relacionada con la relevancia de la ética en el trabajo, el patrón es similar, pero una vez más latinoamericanos dan un mayor porcentaje de alta valoración en la escala, dando más importancia a la ética que sus colegas europeos.

\section{Análisis Factorial}

Como último paso en la comparación entre los graduados de ambos continentes, se analizaron cuestiones relacionadas con su rol (responsabilidad, autonomía, relaciones internas - externas y la ética) mediante análisis factorial exploratorio (AFE). El objetivo es comprobar si los graduados universitarios de cada continente perciben de manera diferente la pertinencia y vinculación entre las diferentes variables. El análisis se realizó para cada continente, mediante el análisis de componentes principales (ACP), para una posterior comparación y análisis de los resultados. 
La fiabilidad de las escalas se comprobó mediante el Alfa de Cronbach, obteniendo valores de 0,759 para los graduados europeos y 0,798 para los graduados de Latinoamérica. Ambos valores indican un buen nivel de fiabilidad de las escalas utilizadas.

En el caso de los graduados europeos, se utilizaron los datos de catorce países europeos, como resultado del $\mathrm{ACP}$, se identificaron tres factores subyacentes, que explican hasta el $61,3 \%$ de la variabilidad total. El valor KMO es 0,762 y la prueba de esfericidad de Bartlett es altamente significativa ( $p>0.000$ ). En la tabla 4 se presenta los resultados del ACP, incluyendo la identificación de los factores y el porcentaje de varianza explicada por cada factor. La columna denominada "componente" corresponde a la identificación de la naturaleza del factor.

\begin{tabular}{|c|c|c|c|}
\hline $\mathbf{N}^{\circ}$ & $\begin{array}{l}\text { Componentes } \\
\text { Interpretados }\end{array}$ & Variables & $\begin{array}{l}\text { \% Varianza } \\
\text { Explicada }\end{array}$ \\
\hline \multirow{4}{*}{1} & \multirow{4}{*}{ Responsabilidad } & $\begin{array}{c}\text { Responsabilidad de establecer } \\
\text { objetivos para la organización }\end{array}$ & \multirow{4}{*}{26,189} \\
\hline & & $\begin{array}{l}\text { Responsabilidad de establecer } \\
\text { objetivos para el propio trabajo }\end{array}$ & \\
\hline & & $\begin{array}{c}\text { Responsabilidad de decidir } \\
\text { estrategias de trabajo para la } \\
\text { organización }\end{array}$ & \\
\hline & & $\begin{array}{c}\text { Responsabilidad de decidir cómo } \\
\text { hacer el trabajo }\end{array}$ & \\
\hline \multirow{4}{*}{2} & \multirow{4}{*}{$\begin{array}{l}\text { Relaciones en el } \\
\text { Trabajo }\end{array}$} & $\begin{array}{l}\text { Los compañeros, clientes, etc. } \\
\text { acuden a mí para pedirme consejo }\end{array}$ & \multirow{4}{*}{23,645} \\
\hline & & $\begin{array}{c}\text { Mantengo informados a mis } \\
\text { compañeros de profesión sobre } \\
\text { nuevos desarrollos en mi campo }\end{array}$ & \\
\hline & & $\begin{array}{c}\text { Suelo iniciar relaciones } \\
\text { profesionales con expertos ajenos a } \\
\text { la organización. }\end{array}$ & \\
\hline & & $\begin{array}{l}\text { Las cuestiones de ética profesional } \\
\text { forman parte importante de mis } \\
\text { funciones. }\end{array}$ & \\
\hline 3 & Perjuicio & $\begin{array}{c}\text { Perjuicio a la organización al } \\
\text { cometer un error grave en el trabajo }\end{array}$ & 11,548 \\
\hline
\end{tabular}

Método de Rotación: Varimax con Normalización Kaiser

Tabla 4. Análisis Factorial - Europa (14 países)

Hay tres factores que tienen una influencia significativa. El primer factor, explica el $26,2 \%$ de la varianza total, incluye cuatro preguntas relacionadas con la responsabilidad y la autonomía. El factor 2, explica el $23,6 \%$ de la varianza total y está formado por las cuestiones relacionadas con las relaciones y la ética. Por último, el factor 3 está formado sólo por la cuestión relacionada con el daño potencial que podría causar a la organización por errores en la toma de decisiones. Es interesante hacer notar que para los graduados europeos, los resultados indican que este nivel de daño es independiente de la responsabilidad que tienen en la organización. La responsabilidad y autonomía son los factores más importantes para los graduados europeos.

Los resultados del análisis por componentes principales realizado a los graduados de Latinoamérica, se muestran en la tabla 5. También se obtuvieron tres importantes factores y explican hasta el $62,3 \%$ de la varianza total. El valor KMO es 0,788 y la prueba de esfericidad de Bartlett es altamente significativa ( $p>0,000)$.

El primer factor, explica el 22,3\% de la varianza total, está formado por las cuestiones relacionadas con las relaciones que se establecen dentro y fuera de la organización y con la ética. Este es el aspecto más relevante para los graduados de LA, seguido inmediatamente por el segundo factor, que explica el $21,2 \%$ de la varianza total e incluyendo tres preguntas relacionadas con la responsabilidad de los profesionales hacia la organización. El tercer factor incluye preguntas relacionadas con la autonomía que el profesional tiene en su trabajo.

\begin{tabular}{|c|c|c|c|}
\hline $\mathbf{N}^{\circ}$ & $\begin{array}{l}\text { Componentes } \\
\text { Interpretados }\end{array}$ & Variables & $\begin{array}{l}\text { Varianza } \\
\text { Explicada }\end{array}$ \\
\hline \multirow{4}{*}{1} & \multirow{4}{*}{$\begin{array}{c}\text { Relaciones en el } \\
\text { Trabajo }\end{array}$} & $\begin{array}{l}\text { Los compañeros, clientes, etc. } \\
\text { acuden a mí para pedirme consejo. }\end{array}$ & \multirow{4}{*}{22,344} \\
\hline & & $\begin{array}{c}\text { Mantengo informados a mis } \\
\text { compañeros de profesión sobre } \\
\text { nuevos desarrollos en mi campo. }\end{array}$ & \\
\hline & & $\begin{array}{c}\text { Suelo iniciar relaciones } \\
\text { profesionales con expertos ajenos } \\
\text { a la organización. }\end{array}$ & \\
\hline & & $\begin{array}{l}\text { Las cuestiones de ética profesional } \\
\text { forman parte importante de mis } \\
\text { funciones. }\end{array}$ & \\
\hline \multirow{3}{*}{2} & \multirow{3}{*}{$\begin{array}{c}\text { Responsabilidad } \\
\text { con la Organización }\end{array}$} & $\begin{array}{l}\text { Responsabilidad de establecer } \\
\text { objetivos para la organización }\end{array}$ & \multirow{3}{*}{21,263} \\
\hline & & $\begin{array}{c}\text { Responsabilidad de decidir } \\
\text { estrategias de trabajo para la } \\
\text { organización }\end{array}$ & \\
\hline & & $\begin{array}{c}\text { Perjuicio a la organización al } \\
\text { cometer un error grave en el } \\
\text { trabajo }\end{array}$ & \\
\hline \multirow{2}{*}{3} & Autonomía / & $\begin{array}{l}\text { Responsabilidad de establecer } \\
\text { objetivos para el propio trabajo }\end{array}$ & \multirow[b]{2}{*}{18,524} \\
\hline & $\begin{array}{l}\text { con el Puesto de } \\
\text { Trabajo }\end{array}$ & $\begin{array}{l}\text { Responsabilidad de decidir como } \\
\text { hacer el trabajo }\end{array}$ & \\
\hline
\end{tabular}

Método de Rotación: Varimax con Normalización Kaiser

Tabla 5. Análisis Factorial - Latinoamérica (10 países)

Los graduados de Latinoamérica consideran que el principal aspecto de su rol está relacionado con sus relaciones con otros profesionales, mientras que en Europa consideran como aspecto principal la responsabilidad y la autonomía. Sin embargo, el factor 
responsabilidad ocupa el segundo lugar en la opinión de los graduados de Latinoamérica, muy cercana al primer factor. Esto es interesante para decir que una de las diferencias que existen tienen que ver con la importancia que los graduados en ambos continentes dan a los diferentes factores, para los Latinoamericanos la responsabilidad y el daño potencial a la organización están vinculadas en el mismo factor, mientras que para los europeos ambas preguntas son independientes. Esta es una diferencia importante en la percepción del rol.

\section{CONCLUSIONES}

En este estudio hemos utilizado una importante base de datos formada por cerca de 40.000 encuestas dirigida a jóvenes profesionales (cinco años después de su graduación) de catorce países de Europa y diez países de América Latina.

Al analizar los puestos de trabajo de estos jóvenes profesionales, hemos observado que el porcentaje más alto ocupa puestos de profesionales/científicos (61\%), seguidos de los técnicos y profesionales de nivel medio $(19,6 \%)$. El 9,5\% del porcentaje global ocupa puestos de dirección, y el resto está trabajando como oficinistas o en otro tipo de puestos de menor cualificación. Estos valores son coherentes con la estructura que se puede esperar en los jóvenes profesionales, en sus primeros años de actividad profesional.

Para ambos grupos (europeos y latinoamericanos) las posiciones más importantes son similares, pero existen algunas diferencias que resultan interesantes de destacar. Los graduados de América Latina. ocupan en mayor proporción puestos ejecutivos (directivos), pero también de empleados de oficina y otras posiciones. Las diferencias en las características socioeconómicas de los graduados en ambos continentes, probablemente, pueden explicar este hecho.

El nivel de correspondencia entre el área de estudio de los graduados y el área de conocimiento requerido por la organización en sus puestos de trabajo tienen distribución similar, con pequeñas diferencias. Una mayoría muy importante de más del $85 \%$ considera que en su trabajo, el área de conocimiento requerida corresponde a su propia área de conocimiento u otra relacionada.

En ambos grupos, los jóvenes profesionales manifiestan de manera distinta su percepción de responsabilidad frente a la organización. Las respuestas de los graduados de América Latina, muestran que tienen altos niveles de responsabilidad, en consonancia con un mayor porcentaje que ocupan puestos de dirección, mientras que para los europeos este nivel es mucho más bajo. En lo que se refiere a la autonomía, ambos grupos muestran un patrón similar de respuesta con ligeras diferencias.
Un alto porcentaje de graduados en ambos continentes trabajan en organizaciones en las que establecen frecuentes contactos y relaciones con otros colegas, tanto dentro como fuera de la organización. Asimismo, la relevancia de la ética en el trabajo es similar para ambos grupos o ligeramente mayor para los graduados de América Latina.

El análisis factorial mejora el reconocimiento de las diferencias en la apreciación de los diferentes aspectos del rol de los de los graduados europeos y latinoamericanos. Diferentes estructuras y diferentes prioridades están en línea con los comentarios anteriores.

Como resumen final, hemos visto importantes $y$ significativas similitudes y diferencias entre los jóvenes profesionales en Europa y América Latina. Los diferentes entornos económicos y socio-culturales producen diferencias, y los graduados adaptan su actividad a estos entornos.

\section{REFERENCIAS}

[1] A. Toffler, The Third Wave, Random House Inc, 1984.

[2] A. Toffler, Powershift, Bantam Books, 1990.

[3] J.L. Carrascosa, Información: De la era industrial a la sociedad de la información. Madrid: Espasa Calpe, 1991.

[4] M. Castells, La era de la información. Madrid: Alianza Editorial, 1997.

[5] J.L. Cebrian, La red, Madrid: Taurus, 1998.

[6] R. Gibson, Preparando el futuro, Barcelona: Gestión, 2000.

[7] B. Echeverría, "Configuración actual de la profesionalidad”, Letras de Deusto, 91 (31), 2001.

[8] G. De La Dehesa, Comprender la Globalización, $2^{\mathrm{a}}$ Edición Madrid: Alianza Editorial, 2007.

[9] M. Mojmir, Globalization: Trends, challenges and opportunities for countries in transition. Vienna: United Nations. Industrial development organization, 2000.

[10] J. González, A. Rodríguez, "Los Cambios en el entorno y sus repercusiones sobre el mercado laboral," Asociación de Escuelas Universitarias de Relaciones Laborales de Andalucía, revista 9, 2002.

[11] J. Allen, and R. Van der Velden (2005). The flexible Professional in the Knowledge Society: Conceptual Framework of The REFLEX Project. Reflex working paper[online]. http://arno.unimaas.nl/show.cgi?fid=17097 
[12] I. Chiavenato, Introducción a la Teoría General de la Administración. México: Mc.Graw Hill, 2006.

[13] A.H.Y. Hon, and R. B. Rensvold, "An interactional perspective on perceived empowerment: the role of personal needs and task context," Int. J. of Human Resource Management, 17, (5), 2006.

[14]N. Fernández, "El desarrollo profesional de los trabajadores como ventaja competitiva de las empresas," Cuadernos de Gestión, vol. 2 (1), 2002.

[15] J-G. Mora, J.M. Carot, y A. Conchado, "Informe resumen del proyecto ALFA PROFLEX en Latinoamérica. Comparativa con el proyecto REFLEX en Europa," Valencia, España, Centro de Gestión de la Calidad y del Cambio, Universitat Politècnica de València, 2010. 